\title{
Systematic review of major considerations of rapid maxillary expansion
}

\begin{abstract}
Introduction: In the context of rapid maxillary expansion, transverse deficiencies are the main predictors of dentofacial deformities with high prevalence in dental practice. In this sense, the techniques of surgically assisted rapid maxillary expansion (SARME) and non-surgically assisted rapid maxillary expansion (NSARME) are used in order to correct maxillary development through palatal disjunction.
\end{abstract}

Objective: to analyze the main considerations of rapid maxillary expansion, showing its main indications, contraindications, types of expanders, as well as the main dental and skeletal changes produced by them. Methods: We searched the databases EBSCO, SCIELO, PubMed / MEDLINE and Academic Google in the period 2003 to 2018. The main descriptors (Mesh Terms) were Rapid expansion of the maxilla, Rapid assisted surgery of the maxilla, Rapid non-surgical maxillary expansion, Circuit breakers and Clinical studies.

Main Finding: The use of the Hyrax expander has become a poorly indicated procedure since the vast majority of patients with maxillary atresia also have other vertical and / or sagittal discrepancies in the bony bases of the face. In this context, however, the rapid expansion of the surgically assisted maxilla presents as a safe and easy procedure to be performed in the office, and is also a low-cost and acceptable procedure for patients. Conclusion: Based on the literary findings, there is still no consensus on which is the best jaw breaker, and it is necessary to increase the number of clinical and randomized studies, with eligibility criteria that can in fact homogenize the participants. In addition, the literature has clearly shown that it is imperative that dental surgeons have the knowledge and clinical experience to be able to indicate the best procedure for each patient, that is, the rapid expansion of the non-surgical or surgical maxilla.

Keywords: rapid maxillary expansion, rapid assisted surgery of the maxilla, rapid non-surgical maxillary expansion, expanders, clinical studies
Volume 9 Issue 4 - 2018

\author{
Ana Júlia Guerra,' Idiberto José Zotarelli \\ Filho, ${ }^{1,2}$ Carlos Alberto Costa Neves \\ Buchala ${ }^{1,2}$ \\ 'University Center North Paulista (UNORP), Brazil \\ ${ }^{2}$ Department of Scientific Production, Unipos, Brazil
}

\begin{abstract}
Correspondence: Idiberto José Zotarelli Filho, Unipos, Post graduate and continuing education, Street Ipiranga, 3460, São José do Rio Preto SP, I5020-040, Brazil,Tel +55(I7) 98I66-6537, Email scientificresearch@unipos.com.br
\end{abstract}

Received: July II, 2018 | Published: July 23, 2018

\section{Introduction}

In the context of rapid maxillary expansion, transverse deficiencies are the main predictors of dentofacial deformities with high prevalence in dental practice. ${ }^{1,2}$ Thus, patients may present with dental crowding, mandibular discrepancies, tooth mobility, multiple dental losses, gingival recessions, respiratory difficulties, repetitive sinusitis, and mouth breathers. In this sense, the techniques of surgically assisted rapid maxillary expansion (SARME) ${ }^{3}$ and non-surgically assisted rapid maxillary expansion (NSARME) ${ }^{2}$ are used in order to correct maxillary development through palatal disjunction. Rapid maxillary expansion (RME) is an efficient technique in the attempt to compensate for the deficient maxillomandibular relationships being initially described by angell 1 (1860) and Haas (1961). ${ }^{1}$ It has as main objective the maxillary disjunction through palatal expanders and, therefore, improves the transversal dimension of patients affected by maxillary deficiencies. ${ }^{4}$ In this context, NSARME is contraindicated in patients with complete bone maturation and, for these cases, surgical rapid maxillary expansion (SARME) is indicated, which is preferable with Hyrax or Haas expander. ${ }^{1}$ The study of maxillary discrepancies is currently an area of great scientific interest in dentistry. SARME can be performed by maxillary osteotomy and rafepalatine associated with a palatal expander, or LeFort I osteotomy associated with maxillary segmentation. ${ }^{4,5}$ From the Haas dental orthodontic expander, many others were developed, including the modified Haas and the Hyrax apparatus. ${ }^{6}$ The latter is a supported dento expander that leads to greater posterior dentoalveolar inclinations, if compared as Haas expander. ${ }^{6}$ In this scenario, the choice of a breaker for maxillary correction is important and can be selected by the main predictors such as bone structure, alveolar processes, the presence or absence of dental elements, the hygiene of the patient, the dentition to be mixed or not, the need for a greater anterior or posterior increase, dentoalveolar inclinations, interdental distances, vertical destabilization and age. ${ }^{1,7}$

Although it is a satisfactory procedure in children and adolescents, the technique of rapid non-surgical expansion presents failures in adult patients, because with skeletal maturation, the response to expansion forces is diminished. The median palatine suture was considered to be the area of greatest resistance to expansion, however, it has been shown that the areas of increased resistance are zygomatotemporal, zygomatrontal and zygomatomaxillary sutures. ${ }^{1}$ Adding to this is the decrease in bone bioplasticity, which makes the maxillary forces dissipating in the maxilla. ${ }^{1}$ Several maxillary osteotomies were developed to expand the maxilla laterally, along with rapidly expanding orthodontic procedures. ${ }^{3,8}$ More recently, it has been shown that only osteotomies in maxillary abutments are sufficient to achieve expansion. ${ }^{3}$ These osteotomies may or may not be complemented by a buccal osteotomy between the roots of the central incisors. ${ }^{8}$ The rapid expansion of the surgically assisted maxilla is an effective procedure but restricts to patients who present only maxillary transverse 
problems. ${ }^{3}$ In cases of deficiencies in other plans, the surgically assisted expansion can be performed as a first surgical time, not excluding the correction of the other plans in a subsequent surgery. Currently, when transverse maxillary deficiency is associated with others, the patient may undergo a single surgical procedure, in which this atresia is corrected with multi-segmentated maxillary osteotomy. ${ }^{3,8}$ The rapid expansion of the surgically assisted maxilla is an effective and safe procedure for the correction of transverse deficiency in adult patients. ${ }^{1,3}$ Among its advantages, we can mention the speed to obtain the expansion, the safety for corrections of up to $14 \mathrm{~mm}$ and the possibility of using local anesthesia, which reduces the cost of the procedure. ${ }^{9}$ A correct diagnosis of the transversal deficiencies of the maxilla and a treatment plan performed jointly by the orthodontist and the maxillofacial surgeon make possible the correct correction of these deficiencies and the satisfaction of the patients. ${ }^{3}$ Therefore, the objective of the present study was to analyze by means of a systematic review of the literature on the main considerations of the rapid expansion of the maxilla, evidencing its main indications, contraindications, types of expanders, as well as the main dental and skeletal alterations by them produced.

\section{Methods}

\section{Study design}

Following the criteria of literary search with the use of the Mesh Terms that were cited in the item below on "Search strategies", a total of 76 papers that were submitted to the eligibility analysis were collated and, after that, 23 studies were selected, following the rules of systematic review-PRISMA (Transparent reporting of systematic reviews and meta-analyses-http://www.prismastatement.org/).

\section{Sources of information}

The review protocol was based on the criteria of literary search with the use of mesh terms in the main databases such as Pubmed, Medline, Bireme, EBSCO, Scielo, etc. All references are registered in EndNote by the site: http://www.myendnoteweb.com/EndNoteWeb. html?cat=myrefs\&

\section{Search strategy}

In general, as an example, the search strategy in MEDLINE / Pubmed, Web Of Science, ScienceDirect Journals (Elsevier), Scopus (Elsevier), OneFile (Gale) followed the following steps: - search for mesh terms: Rapid maxillary expansion. Rapid assisted surgery of the maxilla, Rapid non-surgical maxillary expansion, Circuit breakers, Clinical studies, use of the bouleanos "and" between mesh terms and "or" among historical findings.

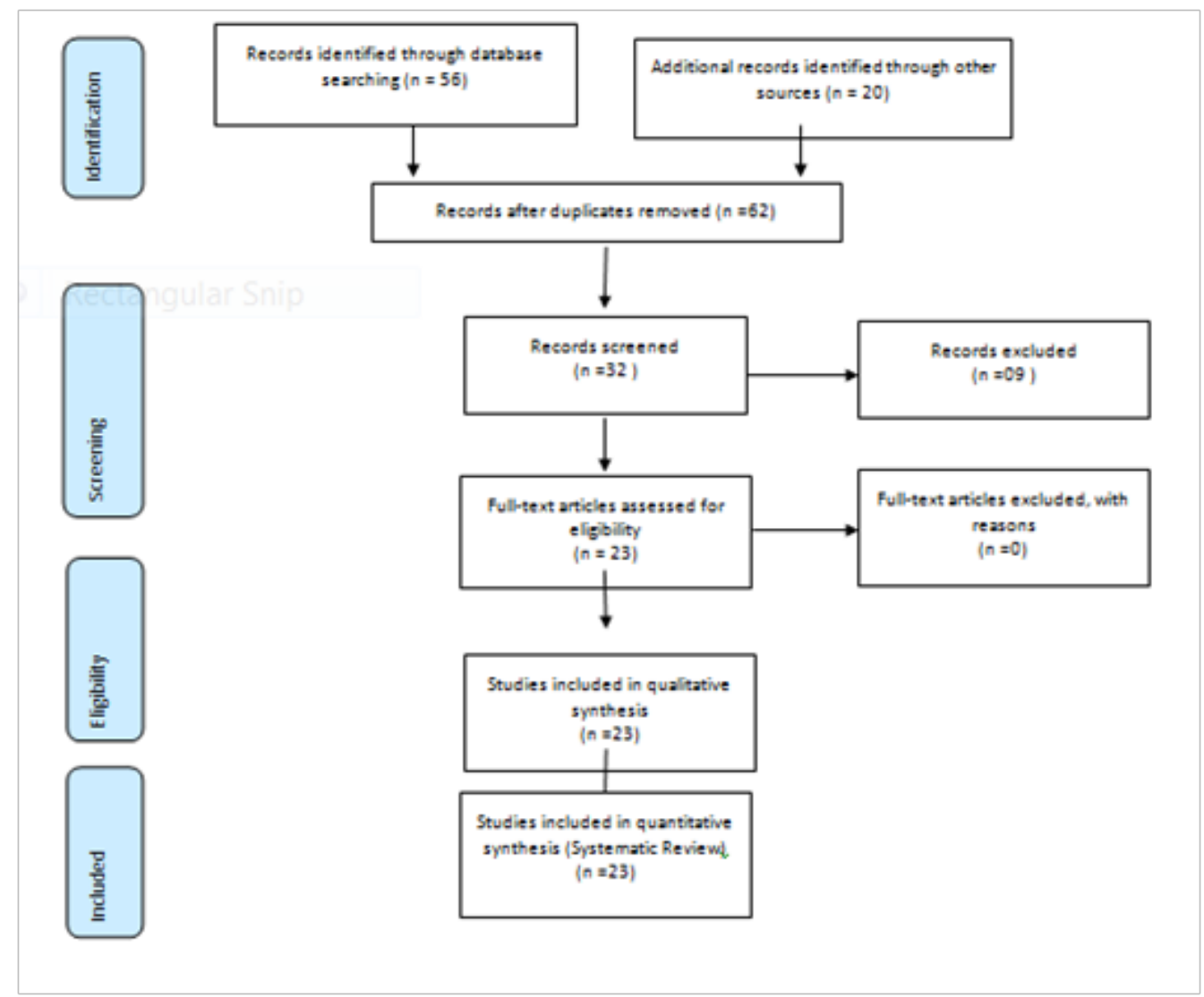




\section{Major findings-development}

According to Table 1, the main considerations regarding the use of SARME and NSARME were listed. The surgical expansion of the maxilla with expander, local anesthesia is used in $100.0 \%$ of clinical cases. ${ }^{1,2}$ Maxillary multi-segmentation solves almost all clinical cases where maxillary atresia is present, allowing the accurate correction of this atresia and correct positioning of the teeth in occlusion, with important postoperative stability. ${ }^{3}$ Thus, the surgical expansion of the maxilla with the use of the Hyrax expander has become a poorly indicated procedure, since the great majority of patients with maxillary atresia also have other vertical and/or sagittal bony bases of the face. ${ }^{4}$ In this context, however, the rapid expansion of the surgically assisted maxilla presents as a safe and easy procedure to be performed in the office, and is also a low-cost and acceptable procedure for patients. Furthermore, the arches that were segmented are also easier to be orthodontically terminated after surgical treatment, to the extent that some experienced orthodontists systematically prefer upper arch segmentation not only to treat it transversally, but also to finalize better and faster clinical cases..$^{1-5}$ In the historical follow-up of the scientific works, the authors Garib et al., ${ }^{10}$ used the Haas and Hyrax expanders in 11 girls between 11 and 14 years of age, divided into two groups and did not observe, for transverse maxillary expansion, representative differences in the two groups; however, the inclination of maxillary premolars was superior in Hyrax expanders.

Table I Major consideration on the SARME and NSARME

\section{Major considerations on the SARME and NSARME}

SARME is indicated for complete bone maturation, transverse deficiencies greater than $5 \mathrm{~mm}$, isolated transverse deformities, transverse deformities associated with vertical and/or anteroposterior deformities, and in transverse maxillary deficits with greater involvement in the anterior region. ${ }^{1,3,9}$

NSARME is indicated for patients with incomplete bone maturation, and there is no consensus in the literature regarding the best age for treatment. ${ }^{3,8}$

The Hyrax expansor are better hygienic and indicated for SARME, while the Haas have better distribution of forces because they have an acrylic covering that rests on the palate."

Both NSARME and SARME improve upper airflow, decreasing respiratory distress, since they promote the increase of the air cavity. 1,9

There is no significant improvement in the results of the expansion when the osteotomy of the pterygoid pillar is performed.'

SARME is more clinically stable when compared to the segmental expansion and is also recommended for clinical cases with marked discrepancy and vestibular slope of molars. ${ }^{1,9}$

The contraindications of this procedure are excessive maxillary prognathism, bimaxillary protrusion, isolated crossbites and patients with excessive vertical development of the face. ${ }^{?}$

Other authors obtained satisfactory results in their study by means of the SARME technique, where they performed a LeFort I osteotomy, separating the pterygoid blades and activation of the Hyrax expander apparatus. ${ }^{8}$ The authors stated that an ERM treatment should take into account the age group of the patient. The areas of bone resistance in the jaw are palatine suture, frontal suture, zygomatic-temporal suture, intermaxillary suture and, mainly, the pterygoid process, where some surgeons opt for separation, since the risk of damage to the pterygoid plexus is large. ${ }^{3}$ In a comparative study with 31 randomly divided patients, where the Group used the Hyrax expander and Group B the Haas expander, Scanavini et al., ${ }^{11}$ found that Hyrax expanders demonstrated a higher risk of relapse. In the sequence, Claro et al., ${ }^{12}$ found significant changes in intercanal and intermolar distances using the Hyrax orthodontic appliance, thus proving a greater posterior cross-sectional increase. Thus, SARME is a safe, easy-toperform technique and the intercurrences are usually contingent. In addition, patients are satisfied. Still, the option for the best surgical technique of SARME is to observe some clinical criteria: transverse deficiencies greater than 5mm; isolated transverse deformities; transverse deformities associated with vertical and/or anteroposterior deformities and transverse maxillary deficits with greater involvement in the anterior region. ${ }^{1,3,8}$ A systematic review work showed the results of some studies that used the rapid surgical expansion technique associated with a Hyrax maxillary expander in 20 patients between 15 and 54 years, obtaining a satisfactory mean final result. ${ }^{1}$ In addition, other authors used the Hyrax and Haas appliances in 62 patients who needed rapid maxillary expansion and observed greater expansion at the first molars and maxillary premolars crowns in Hyrax appliances. ${ }^{1}$

In the study by Rossi et al., ${ }^{13}$ the vertical and anteroposterior effects of rapid maxillary expansion were evaluated cephalometrically using a study of 26 children, 14 females and 12 males, with a mean age of 8.7 years, presented posterior crossbite and maxillary narrowing. A McNamara type device made of clear acrylic resin covering the posterior teeth was used, and an expander screw located on the palatine raphe between the second deciduous molars. The rapid expansion of the maxilla using a breaker with occlusal acrylic cover did not promote vertical cephalometric alterations and sagittal lesions. The vertical changes were small and transient. The work of Melgaço et al. ${ }^{14}$ was analyzed in 31 patients with Angle Class I malocclusion, transverse palatal and lingual changes in patients submitted to rapid maxillary expansion. The sample was divided into 2 groups, Group 1 consisting of 17 patients treated with the Haas expander and Group 2, by 14 patients treated with Hyrax. No statistically significant differences were found when comparing the Haas and Hyrax devices. In the study by Baratieri et al., ${ }^{15}$ with the objective of evaluating by means of computed tomography the transversal dimensions of the nasomaxillary complex of patients submitted to rapid maxillary expansion, performed a study in 30,18 of the male gender and 12 of the female. The patients were divided into two groups, and the control group consisted of 10 boys and 5 girls with a mean age ranging from 9 years to 4 months. The treated group consisted of patients who needed maxillary expansion, composed of 8 boys and 7 girls, with a mean age of 9 years and 6 months, who underwent Haas expander treatment. Was Haas and the activation protocol proposed by Haas and at the time of installation of the activation of a complete revolution $(0.8 \mathrm{~mm})$, followed by daily activations of $1 / 4$ back in the morning and $1 / 4$ back in the evening. Therefore, there was a significant increase in the widths between the molars, maxillaries, palatal and nasal in relation to the control group.

Woller et al., ${ }^{16}$ evaluated by computer tomography the changes that occur in the maxillary sutures in children submitted to rapid expansion of the maxilla treated with a Hyrax-type device, carried out a study in 25 patients (10 men, 15 women) who presented discrepancies of the maxilla. The exclusion criteria selected were patients with craniofacial anomalies, including cleft lip and palate, and patients 
using orthodontic appliances prior to initiation of treatment with rapid maxillary expansion. Each patient was scanned twice: T0 and T1. The first image (T0) was obtained before the expander was installed, and the second image (T1) obtained after the last activation of the device. Thus, there was a large displacement of the medial palatine suture, but this displacement also occurs in other sutures, such as frontonasal, intermaxillary and zygomaticomaxillary. Izuka et al., ${ }^{9}$ analyzed the short-term effects of rapid maxillary expansion on the upper airway dimensions, as well as evaluating the impact of this therapy on quality of life, by means of computed tomography studies, performed a study on 25 respirator patients with 14 women and 11 men. Standardized questionnaire was performed with the parents before and after the rapid maxillary expansion, aiming to evaluate if there was improvement in patients' quality of life. CT scans were performed prior to treatment, and then modified Hyrax-type appliances were installed. Therefore, rapid maxillary expansion promoted a significant improvement in the quality of life of mouth breathing patients with maxillary atresia.

\section{Discussion}

Based on the literature findings, rapid maxillary expansion is a feature used in orthodontic practice, where the middle palatine suture is opened and the craniofacial complex sutures are disorganized. ${ }^{1,2}$ However, it is not always possible to achieve its opening, since, at the end of facial growth, its ossification occurs. ${ }^{9}$ Therefore, the closure of the medial palatine suture is determinant in situations of failure of rapid maxillary expansion.

\section{NSARME indications and contraindications}

The procedure of rapid expansion of the maxilla by the opening of the medial palatine suture is indicated in the treatment of malocclusions with real and relative maxillary deficiency; of chronic nasal disability who exhibit nasal respiratory problems and with problems related to arch length. ${ }^{1}$ As contraindications, excessive maxillary prognathism, bimaxillary protrusion, isolated crossbites and patients with excessive vertical development of the face are mentioned. ${ }^{1,2}$ In order to promote the opening of the medial palatine suture, there are several breaker devices; the most used being the dentifrice holder (Haas), the dentosupport (Hyrax), and the dentosupport with occlusal acrylic cover. ${ }^{1}$ Thus, as a literary corollary, the NSARME technique is adequate for patients with incomplete maxillary bone maturation and transverse maxillary deficiency that cannot be corrected by fixed apparatus., ${ }^{4,5}$ Another indirect benefit of maxillary expansion techniques is the improvement of upper airflow reducing respiratory distress, promoting the increase of air cavity. ${ }^{5}$ Thus, the most accepted age for performing NSARME varies widely according to the literature, being proposed for patients with a maximum age of 14 years in women and 16 years in men, according to. ${ }^{6}$ Some authors were successful with the age range between 15 and 54 years, using the NSARME technique with a Haas expander in a 19-year-old patient, contradicting the authors who affirmed the efficacy of the technique only in patients with incomplete bone maturation. ${ }^{1,9}$ Therefore, there is no consensus in the literature regarding a fixed age for NSARME indication. Still, there is an ineffectiveness of this technique in adult patients who have already surpassed the peak of growth. In addition to bone consolidation as a limiting factor. In addition, Rossi et al., ${ }^{13}$ stated that the NSARME technique is contraindicated in cases of multiple dental absence, large dentoalveolar inclinations for vestibular, gingival recession, alveolar bone loss, posterior dental mobility and in adult patients with advanced skeletal maturation.

\section{SARME indications and contraindications}

SARME is indicated for patients with more advanced age and consequent complete bone maturation, for transverse deficiencies greater than $5.0 \mathrm{~mm}$, isolated transverse deformities, transverse deformities associated with vertical and/or anteroposterior deformities, and in transverse maxillary deficits with greater involvement in the region previous. ${ }^{1,3,8}$ In this sense, transverse deficiencies should be analyzed through clinical examination, analysis of gypsum models, cephalometric and occlusal radiographs in an attempt to visualize prevalent alterations such as anterior and posterior cross bites, dentoalveolar inclinations, lack of dental elements, prognathism or retrognathism, gingival recession, alveolar bone loss, posterior dental mobility and skeletal maturation, in order to select the appropriate maxillary expansion technique. ${ }^{3,8}$

\section{Major considerations of ideal expander}

The modified Haas circuit breaker produces a lower dentoalveolar slope than the Hyrax appliances, in addition to increasing the interdental distance between the canines ${ }^{1,9}$ Also, the acrylic cover in the Haas expander directs the force vector to the center of resistance of the maxilla, increasing the transverse dimension, not affecting the sagittal dimension, besides not provoking dental orthodontic movement. ${ }^{9}$ The Hyrax appliances have a higher interdental increase between the molars and premolars and a posterior transverse increase, according to Garib et al., ${ }^{10}$ and Claro et al., ${ }^{12}$ Other authors, however, did not observe significant clinical differences between Haas and Hyrax. ${ }^{1}$ However, Kilic et al. ${ }^{17}$ indicates the use of the modified Haas circuit breakers as it directs its tensile force to the center of the maxilla, not affecting both the inclination of the teeth. In addition, the literature states that the use of Hyrax circuit breakers presents a higher risk of recurrence when compared to Haas.

\section{Conclusion}

Based on the literary findings, there is still no consensus on which is the best jaw breaker, and it is necessary to increase the number of clinical and randomized studies, with eligibility criteria that can in fact homogenize the participants. In addition, the literature has clearly shown that it is imperative that dental surgeons have the knowledge and clinical experience to be able to indicate the best procedure for each patient, that is, the rapid expansion of the non-surgical or surgical maxilla. ${ }^{18-24}$

\section{Acknowledgements}

None.

\section{Conflict of interest}

The author declares that there is no conflict of interest.

\section{References}

1. Davoudi A, Amrolahi M, Khaki H. Effects of laser Therapy on patients who underwent rapid maxillary expansion; a systematic review. Lasers Med Sci. 2018;33(6):1387-1395.

2. Wang MH, Ge ZL, Tian L, et al. Effect of three types of rapid maxillary expansion: a three-dimensional finite element study. Zhonghua Kou Qiang Yi Xue Za Zhi. 2017;52(11):678-683.

3. Huizinga MP, Meulstee JW, Dijkstra PU, et al. Bone-borne surgically assisted rapid maxillary expansion: A retrospective three-dimensional evaluation of the asymmetry in expansion. J Craniomaxillofac Surg. 


\section{8;S1010-5182(18):30243-30249.}

4. Ghoussoub MS, Rifai K, Garcia R, et al. Effect of Rapid Maxillary Expansion on Glenoid Fossa and Condyle-Fossa Relationship in Growing Patients (MEGP): Study Protocol for a Controlled Clinical Trial. J Int Soc Prev Community Dent. 2018;8(2):130-136.

5. Lombardo L, Carlucci A, Maino BG, et al. Class III malocclusion and bilateral cross-bite in an adult patient treated with miniscrew-assisted rapid palatal expander and aligners. Angle Orthod. 2018.

6. Lione R, Brunelli V, Franchi L, et al. Mandibular response after rapid maxillary expansion in class II growing patients: a pilot randomized controlled trial. Prog Orthod. 2017;18(1):36.

7. Bell RA. A review of maxillary expansion in relation to rate of expansion and petient's age. Am J Orthod. 1982;81(1):32-37.

8. Nikolaev AV, Andreishchev AR, Kutukova SI. Comparative biomechanical study of surgically assisted rapid palatal expansion with tooth-borne and bone-borne expanders. Stomatologiia (Mosk). 2017;96(5):48-55.

9. Izuka EN, Feres MFN, Pignatari SSN. Immediate impact of rapid maxillary expansion on upper airway dimensions and on the quality of life of mouth breathers. Dental Press J Orthod. 2015;20(3):43-49.

10. Garib DG, Menezes MHO, Silva Filho OG, et al. Immediate periodontal bone plate changes induced by rapid maxillary expansion in the early mixed dentition: CT findings. Dental Press J Orthod. 2014;19(3):36-43.

11. Scanavini MA, Reis SAB, Simões MM, et al. Avaliação comparativa dos efeitos maxilares da expansão rápida da maxila com os aparelhos de Haas e Hyrax. Rev Dent Press Ortodon Ortop Facial. 2006;11(1):60-71.

12. Claro CAA, Abrão J, Reis SAB, et al. Correlation between transverse expansion and increase in the upper arch perimeter after rapidmaxillary expansion. Braz Oral Res. 2006;20(1):76-81.

13. Rossi M, Rossi A, Abrão J. Skeletal alterations associated with the use of bonded rapid maxillary expansion appliance. Braz Dent J. 2011;22(4):334-339.
14. Melgaço CA, Neto JC, Jurach EM, et al. Rapid maxillary expansion effects: An alternative assessment method by means of cone-beam tomography. Dental Press J Orthod. 2013;19(5):88-96.

15. Baratieri C, Nojima LI, Alves JRM, et al. Efeitos transversais da expansão rápida da maxila em pacientes com má oclusão de Classe II: avaliação por Tomografia Computadorizada Cone Beam. Dental Press J Orthod. 2010;15(5):89-97.

16. Woller JL, Kim KB, Behrents RG, et al. An assessment of the maxilla after rapid maxillary expansion using cone beam computed tomography in growing children. Dental Press J Orthod. 2014;19(1):26-35.

17. Kilic N, Kiki A, Oktay H, et al. Effects of rapid maxillary expansion on conductive hearing loss. Angle Orthod. 2008;78(3):409-414.

18. Albuquerque RR, Eto LF. Previsibilidade de sucesso na disjunção palatina avaliada pelo estágio de maturação esquelética. Rev Dental Press Ortodon Ortop Facial. 2006;11(2):74-83.

19. Alpiner ML, Beaver HA. Criteria for rapid maxillary expansion. J Mich Dent Ass. 1971;53(2):39-42.

20. Janson G, Tompson BD, Donald GW, et al. Estudo cefalométrico das alterações dento-esqueléticas decorrentes da expansão rápida da maxila com ancoragem esquelética. $R$ Dental Press Ortodon Ortop Facial. 2008;13(1):124-140.

21. Baratieri CL, Alves JRM, Mattos CT, et al. Transverse effects on the nasomaxillary complex one year after rapid maxillary expansion as the only intervention: A controlled study. Dental Press J Orthod. 2014;19(5):79-87.

22. Barreto GM, Gandini JRLG, Raveli DB, et al. Avaliação transversal e vertical da maxila, após expansão rápida, utilizando um método de padronização das radiografias póstero-anteriores. $R$ Dental Press Ortodon Ortop Facial. 2005;10(6):91-102.

23. Biedercmn W. A hygienic appliance for rapid expansion. J Pract Orthod. 1968;2(2):67-70.

24. Kurt G, Altuğ AT, Türker G, et al. Effects of Surgical and Nonsurgical Rapid Maxillary Expansion on Palatal Structures. J Craniofac Surg. 2017;28(3):775-780. 